over two million hospitalized patients develop severe adverse drug reactions in the United States annually, incurring a direct medical cost of 200 billion US dollars (USD). This indicates a huge potential in reducing healthcare costs even if only a small proportion of adverse drug reactions are preventable by genotype-guided prescription. The current clinical applications of pharmacogenetics are mostly limited to reactive pharmacogenetic testing, in which investigations are ordered only when certain high-risk medications are prescribed, or after an adverse drug reaction has occurred. In contrast, preemptive pharmacogenetic testing allows the optimization of dosing based on genotype information at the time of prescription, minimizing the risk of undesired outcomes.

Objectives Pharmacogenetics (PGx) encompasses the potential to improve therapeutic response and reduce adverse drug reaction in the era of precision medicine. However, Chinese PGx data is limited. To address this issue, we examined the spectrum of 133 actionable pharmacogenetic variants and rare deleterious variants in 108 pharmacogenes and estimated the proportion of dispensed drugs that may potentially benefit from genotype-guided prescriptionusing an exome sequencing (ES) cohort.

Methods Secondary analysis of ES data was conducted to study pharmacogenetics in $1116 \mathrm{HK}$ Chinese, which included 622 males and 494 females. The projected preemptive pharmacogenetic testing prescription impact was evaluated based on the patient prescription data between January 1, 2019 and December 31, 2019 in the HK public healthcare system, serving 7.5 million people and accounting for approximately $90 \%$ of all secondary and tertiary healthcare services provided.

Results Twenty-nine actionable pharmacogenetic variants/alleles were identified in our cohort. Nearly all (99.6\%) subjects carried at least one actionable pharmacogenetic variant, with a median of four variants, whereas $93.5 \%$ of subjects harbored at least one rare deleterious pharmacogenetic variant, with a median of two variants. The frequency of actionable genotypes was the highest in recipients of clopidogrel (57.21\%), tacrolimus (43.38\%), and warfarin (43.13\%). Based on the prescription data of the public healthcare system in 2019, $13.4 \%$ of the HK population was prescribed with drugs with implicated actionable pharmacogenotypes in the HK population. The total expenditure on actionable drugs was $33,520,000$ USD, and it was estimated that $8,219,000$ USD $(24.5 \%)$ worth of drugs were prescribed to patients with an implicated actionable genotype.

Conclusions In conclusion, nearly all individuals carried at least one actionable pharmacogenetic variant and one rare, deleterious pharmacogenetic variant in our cohort. It was estimated that oneseventh of the $\mathrm{HK}$ population received at least one of the 36 drugs with CPIC guideline recommendations, and 8,219,000 USD worth of drugs were prescribed to patients with an implicated actionable genotype. The results showed that secondary use of ES data is feasible for pharmacogenetic analysis, and preemptive pharmacogenetic testing has the potential to support prescription decisions in inpatient and specialist outpatient settings in the HK Chinese population.

\section{PONDERAL INDEX IN LOW BIRTH WEIGHT BABIES}

Rydam Basnet, Sunil Raja Manandhar, Rajan Phuyal, Ritika Basnet. Nepal
Background Low birth weight is a common problem in neonates. The ponderal index (PI) is a simple anthropometric measurement that is used to diagnose impaired fetal growth. $\mathrm{PI}=$ Weight $(\mathrm{GM}) /$ Length $(\mathrm{CM}) 3 \times 100$.

The value of ponderal index $<2.0$ between 29 and 37 weeks and $<2.25$ beyond 37 weeks are indicative of intrauterine fetal malnutrition, and the babies are classified as disproportionate IUGR. Low ponderal index or disproportionate Intra uterine growth retardation (IUGR) has higher neonatal morbidity than their proportionate counterparts.

Objectives To determine the ponderal index of the low birth weight babies and to classify them accordingly.

Methods A descriptive cross sectional study which was carried out in Paediatrics Department of Kathmandu Medical College Teaching Hospital from July 2020 to September 2020. Ethical clearance was taken from the Institutional Review Committee.

The study population consisted of low birth weight neonates on any period of gestation. Informed written consent was taken from the parents. Babies with congenital malformations were excluded.

The sample size was 212 neonates. The eligible babies were examined after birth. Birth weight and the length were taken by standard weighing machine and infantometer. For perinatal factors, interview of parents were taken using the designed proforma.

Results A total of 210 low birth weight babies fulfilling the inclusion criteria were enrolled in this study.105 (49.5\%) mothers were primiparous. $116(54.7 \%)$ babies were born vaginally. $111(52.4 \%)$ babies were males.

Abstract 109 Table 1 Classification according to gestational age

\begin{tabular}{lll}
\hline Gestational age (in weeks) & Number of babies & Percentage (\%) \\
\hline $28-32$ weeks & 17 & 8 \\
$33-36$ weeks & 94 & 44.3 \\
$37-39$ weeks & 94 & 44.3 \\
$40-42$ weeks & 7 & 3.4 \\
\hline
\end{tabular}

Abstract 109 Table 2 Classification according to birth weight and gestational age based centile chart

\begin{tabular}{llll}
\hline & Centiles & $\begin{array}{l}\text { Number of } \\
\text { babies }\end{array}$ & $\begin{array}{l}\text { Percentage } \\
(\%)\end{array}$ \\
\hline Small for Gestational age (SGA) & $<10$ centile & 122 & 57.5 \\
Appropriate for Gestational age (AGA) & $10-90$ centile & 86 & 40.1 \\
Large for Gestational age (LGA) & $>90$ centile & 4 & 1.9 \\
\hline
\end{tabular}

Abstract 109 Table 3 Classification according to Ponderal index centile chart

\begin{tabular}{lll}
\hline Centile of Ponderal index & Number of babies & $\begin{array}{l}\text { Percentage of } \\
\text { Newborns (\%) }\end{array}$ \\
\hline$<10$ centile & 123 & 58 \\
$10-50$ centile & 52 & 24.5 \\
$51-90$ centile & 24 & 11.3 \\
$>90$ centile & 13 & 6.1 \\
\hline
\end{tabular}


A total of $111(52.3 \%)$ babies were premature. The mean birth weight of the babies was 2052 \pm 352 gms (1050-2490 gms) and the mean gestational age was $35 \pm 2$ weeks (28-42 weeks).

Out of 212 babies, ninety-nine babies (46.7\%) had a low ponderal index $(<2.2$ in term babies, $<2.0$ in preterms $)$ and by definition had disproportionate IUGR, out of which 60 (59.4\%) babies were term and 39 (35.1\%) were preterms.

170 babies $(80.2 \%)$ had no postnatal complications, 15 babies had neonatal sepsis, 10 babies had respiratory distress syndrome, 6 babies had hypoxic-ischemic encephalopathy (4 babies stage I HIE, 2 babies Stage II HIE), 8 babies had transient tachypnea of newborn, 3 babies had hypoglycemia and 2 babies had polycythemia.

Conclusions Ponderal index is a simple and effective tool to identify wasting. In this study, nearly $46.7 \%$ of the low birth weight babies were disproportionate at birth.

\section{PARENTAL DECISION MAKING WHEN CHOOSING A PAEDIATRIC PRIMARY CARE SERVICE: WHAT FACTORS MATTERED MOST?}

Erwin Jiayuan Khoo, Colette Teng Wee, Kurubaran Ganasegeran. Malaysia

\subsection{6/bmjpo-2021-RCPCH.63}

Background In Malaysia, patients are free to choose their own primary care healthcare providers at their own expense. Studies show that patients who choose their own doctor are associated with increased patient satisfaction. Primary care paediatricians realise the need to involve parents as stakeholders in today's competitive healthcare industry.

Objectives This study aimed to identify what factors matter to parents when choosing a paediatric primary care for their children in a free market setting where they have direct access to paediatricians. It also explored if differences in these perceived factors exists between parental education and household income levels.

Methods An analytical cross-sectional study was conducted at two paediatric primary care centres in urban Malaysia. Parents who brought their child to seek treatment at the centres were invited to participate. A face and content validated questionnaire that includes respondents' demographics was devised from the literature and a focus group discussion. A 5-point Likert scale was applied to 25 elements that were organised into five major domains: 'consultations' (CO), 'facilities and services' (FS), 'fees and charges' (FC), 'social media engagement' (SM) and 'doctor's 'appearance' (DA). Elements within each domain were ranked according to a mean ratings score. Internal consistency for each domain was yielded through a Cronbach's alpha $(\alpha)$ value. Independent t-test was used to determine the associations between outcomes and independent variables.

Results 387 parents completed the questionnaires (response rate, 91.3\%). The mean age of the parents was 37.7 years. $295(76.2 \%)$ parents attained a tertiary education. 157 $(40.6 \%)$ parents were bottom tier income earners. Parents rated the provision of FS highest (mean rating score $=41.63$, $\alpha=0.84$ ), followed by ratings on the interaction with healthcare providers during $\mathrm{CO}(34.73,0.77)$. Younger aged parents $(p=0.021)$ and parents from lower household income level $(p=0.001)$ had higher FS rating score compared with older parents and parents of higher income respectively. The top elements in the FS domain were a 'strategic location of the primary care' and 'comfortable and clean waiting room'. The $\mathrm{CO}$ ratings have a predilection towards technical quality, interpersonal manners and successful provider-patient communications during clinical encounters. In exchange, for FC (11.80, $0.75)$, parents appeared to tolerate costlier consultation and treatment, but still appreciate bills that are transparent. While parents with a higher level of education prefer to participate in shared decision-making with the paediatricians $(p=0.002)$, parents from lower household income level rated a significantly higher score in SM $(p<0.001)$ and DA $(p=0.019)$.

Conclusions Where parents are free to choose their children's paediatric primary care service, convenient accessibility and perceived consultation-related proficiency mattered most to parents when deciding. The extent to what makes a difference, appeared to be influenced by parental age, educational and income level. These perceived differences largely confirm the need to invest in the development of individualised support services in a consumer-driven health care system. These preferred elements can provide clue to determine parental satisfaction towards health services provided and predict complex health seeking behaviours.

\section{ASSOCIATION BETWEEN DEGREE OF SENSITIVITY TO INDOOR ALLERGENS AND ASTHMA SEVERITY}

Wai Mar Tin. Myanmar

\subsection{6/bmjpo-2021-RCPCH.64}

Background Aeroallergens, especially indoor aeroallergens have a major role in the pathogenesis and severity of asthma. In Myanmar, data about allergen sensitivity and asthma is still lacking. Understanding the prevalence of the most common aeroallergens in local area can help in the diagnosis and management of asthma.

Objectives

1. To determine the prevalence of sensitivity of indoor allergens in children with asthma

2. To analyze the association between degree of sensitivity to indoor allergens and asthma severity.

Methods This hospital based cross sectional analytical study was conducted in 120 children (aged 6-12 years) with asthma who attended asthma clinic in Yangon Children Hospital. Severity of asthma was assessed by taking history, physical examination and lung function test. It was classified into mild asthma and moderate to severe asthma groups according to the working definition. Skin prick test with eight indoor aeroallergens extracts was performed in 120 children. The degree of the allergic sensitization was assessed by the number of positive skin prick tests and by skin wheal sizes. Associations between degree of atopic sensitization and severity of asthma, were analyzed by using logistic regression

Results The majority of subjects $(67.5 \%)$ had moderate- severe asthma and $32.5 \%$ had mild asthma. $92.5 \%$ of study population had skin prick test positive to at least one indoor allergen. Mono sensitization to aeroallergen was observed in only $11.7 \%$ of patients and the rest were sensitized to more than one allergen among sensitized children. The most prevalent allergen was HDM (Dermatophagoides pteronyssinus and Dermatophagoides farinae) with $64.2 \%$ and $68.3 \%$ respectively. Prevalence of sensitization to other indoor allergens was as follow: Aspergillus fumigatus (40.8\%), Alternaria alternata 